


Science

\title{
INTRODUCING ANTIOXIDANTS AS ESSENTIAL FOR THE MAINTENANCE OF TISSUE LIFE AS DEMONSTRATED IN HUMAN HAIR FOLLICLES
}

\author{
Abraham A Embi Bs *1
}

\begin{abstract}
Hair Follicles have been described as mini-organs having a wide range of functions and relying in tightly regulated molecular signaling for its metabolism, and extensively described to emit biomagnetic fields (BMFs). The ubiquitous enzyme catalase is essential for the eukaryotic cells to maintain toxic Reactive Oxygen Species (such as H2O2) in equilibrium. This manuscript presents a novel optical microscopy technique that utilizes a glass slide sandwich (SDW) where drops of fresh human blood are trapped. Drops of water are placed on the top slide of the SDW, in which a freshly plucked human hair follicle is immersed. In this experimental model, is shown that in water immersed follicles, BMFs interact with the intrinsic BMFs of the blood, this in turn causing vertically detected spatial images resembling two different magnetic profiles (MPs). When hairs are immersed in $35 \% \mathrm{H} 2 \mathrm{O} 2$ oxygen bubbles (O2) are displayed due to the $\mathrm{H} 2 \mathrm{O} 2$ breakdown by the enzyme catalase. When the $\mathrm{O} 2$ breakdown ceases is theorized that tissue death has occurred. Results are presented of images and video-recordings correlating the absence of catalase with cessation of MPs.
\end{abstract}

Keywords: Magnetic Profiles; Cyclotron Resonance; Lorentz Forces; Definition Death; Microscopy; Human Blood; Antioxidants.

Cite This Article: Abraham A Embi Bs. (2018). "INTRODUCING ANTIOXIDANTS AS ESSENTIAL FOR THE MAINTENANCE OF TISSUE LIFE AS DEMONSTRATED IN HUMAN HAIR FOLLICLES." International Journal of Research - Granthaalayah, 6(7), 263-271. https://doi.org/10.29121/granthaalayah.v6.i7.2018.1305.

\section{Introduction}

The present paradigm on the causes defines tissue death as: Cell death is the event of a biological cell ceasing to carry out its functions. The causes range from necrosis to Programmed Cell Death. For the experiments, the hair follicle was selected since it has been classified as a mini-organ (1). This manuscript demonstrates via experiments in vitro that catalase depletion is a main factor in the hair follicle death. In the absence of catalase, cell respiration ceases, thus causing the absence of biomagnetic fieds induced magnetic profiles (MPs). This Biophysical mechanism in being introduced and hypothesized as a marker for cell death. 


\section{Brief History of Electromagnetic Forces Detection in Living Tissue}

In the past, researchers have used highly complex and expensive equipment aiming at the detection of electromagnetic fields in human organs such as the brain and heart $(2,3,4,5)$. Also in that era, a paper published in 1980 introduced "magnetic fields produced by naturally occurring steady currents in the body by using a new magnetic gradiometer in a magnetically shielded room..."finding most of the field over the head is produced by electrical sources associated with the hair follicles of the scalp; this field is produced only as a response to touching or pressing the scalp, in regions where the hair is dense" (6). Three decades later, the findings were duplicated with the introduction of a novel optical microscopy technique using Prussian Blue Stain (PBS) mixed with fine iron particles (7). This novel approach has produced numerous papers confirming the inherent biomagnetism of the human hair, especially biomagnetic fields (BMFs) expressed as spontaneous light rays discharges arising from the hair follicle (Figure $1+$ supplementary video) (8) Recently, and of relevancy to this manuscript, is the penetration of the hair follicles BMFs through glass barriers (9), as well as the in vivo external detectability of sub dermal follicles (10), or fronted hair follicles that were documented interacting with their counterparts (11). Seminal papers published 80 years ago by Pauling and Coryell in PNAS "described in detail the magnetic properties of oxy- and deoxyhemoglobin, as well as those of closely related compounds containing hemes" (12).

\section{Materials and Methods}

By using a simple slide assembly, utilizing a small sterile needle blood drops from the author were smeared on a $25 \times 75 \times 1 \mathrm{~mm}$ glass slide (Globe Scientific \#1301) and sandwiched by a second similar slide, thus forming a sandwich like structure (SDW) (Fig 1).

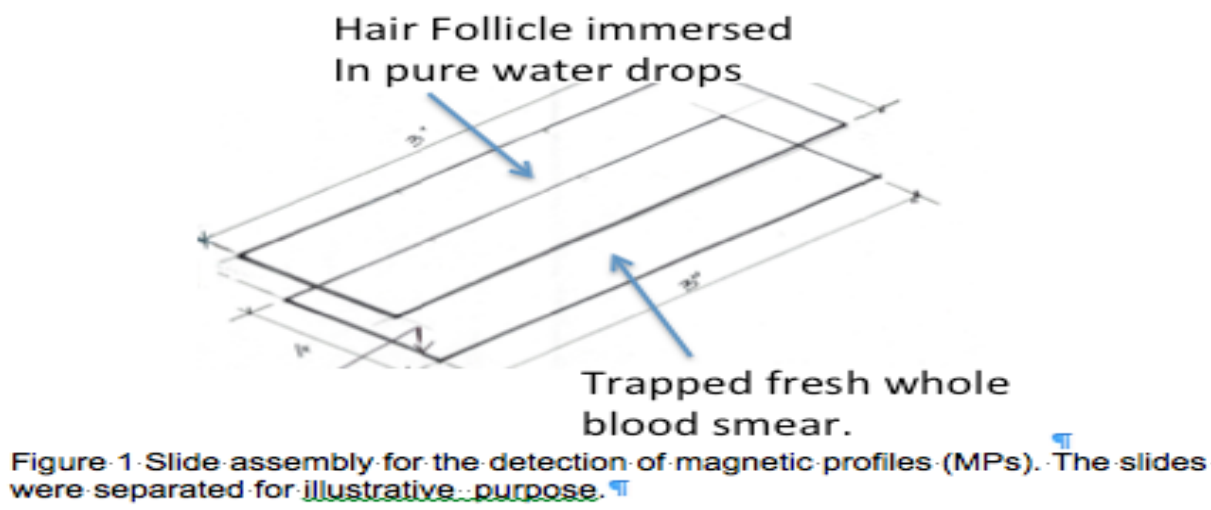

\section{Baseline Recordings}

On the surface of the SDW top slide (Fig 1), a freshly plucked scalp human hairs was placed, fronted and covered by drops of deionized water (resistivity $18.2 \mathrm{M} \Omega . \mathrm{cm}$ ). Recording equipment used was a video microscope (Celestron LCD Digital Microscope II model \#44341 and images downloaded into an Apple System Photo Application.

As the trapped interstitial blood equilibrated in the SDW, a continuous flow of red blood cells ensued and is temporarily seen. At this point a video recording was done $(n=4)$ documented what is theorized to be a human blood/hair follicle MPs interaction (Fig 2). 
The slides of all the experiments $n=10$ were viewed and recorded in a video-microscope, the follicle is focused and the depth of field knob is slowly moved inwards. The first magnetic profiles images appeared, which were seen as having a curved cyclotron resonance pattern. As the knob continuous inwards a second magnetic profile image appears; this time is identified as linear Lorentz Forces as result of the Hall effect (Fig 1A \& 1B)

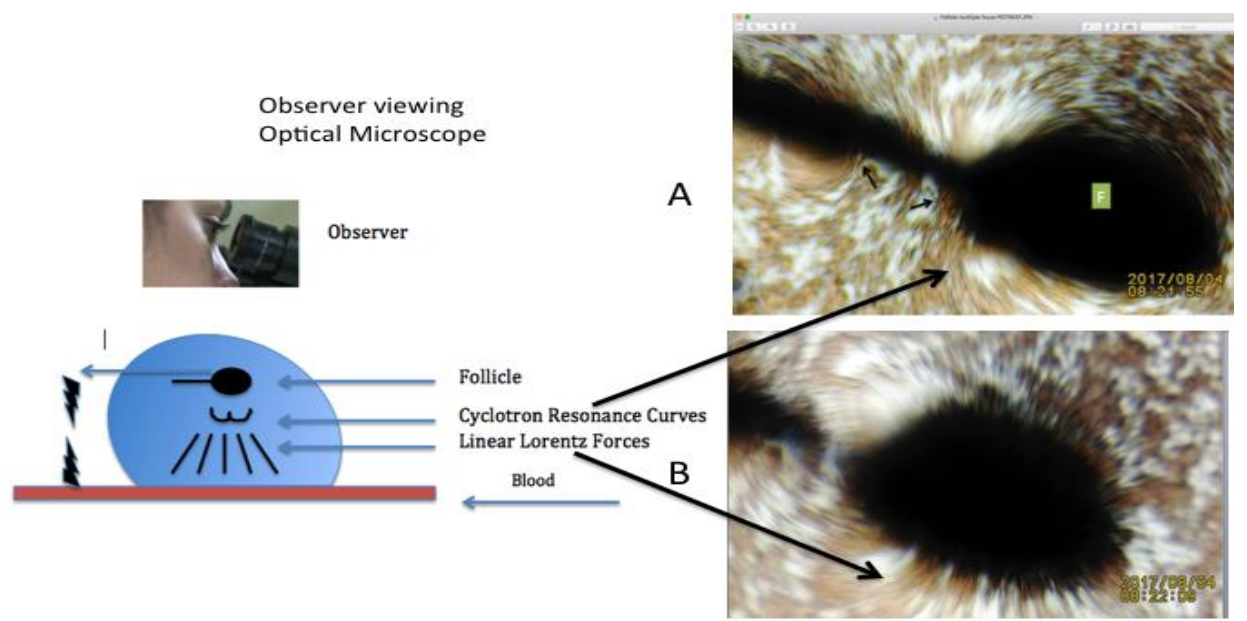

Figure 1A: Display of vertical spatially separated displays of magnetic profiles (MPs). When hair follicle is in slide assembly and observer changes the focusing knob inwards. $A=$ Displayed first are Cyclotron Resonance circumferential curves. $\mathrm{B}=$ When knob turns more inwards, Linear Lorentz Forces are displayed.

A
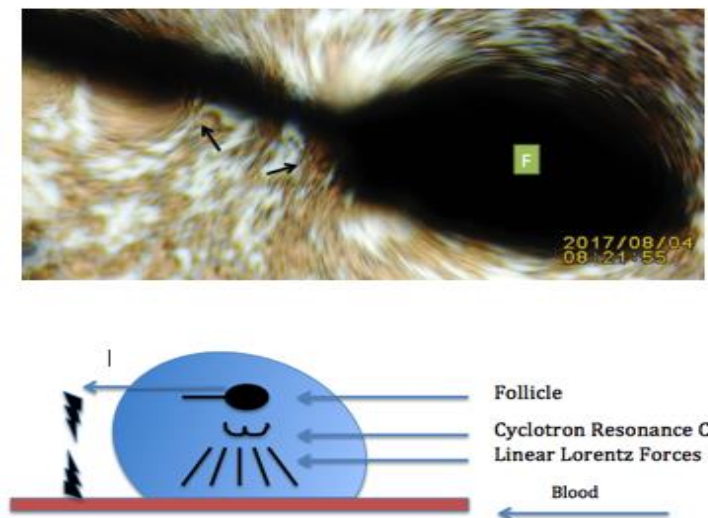

C
B
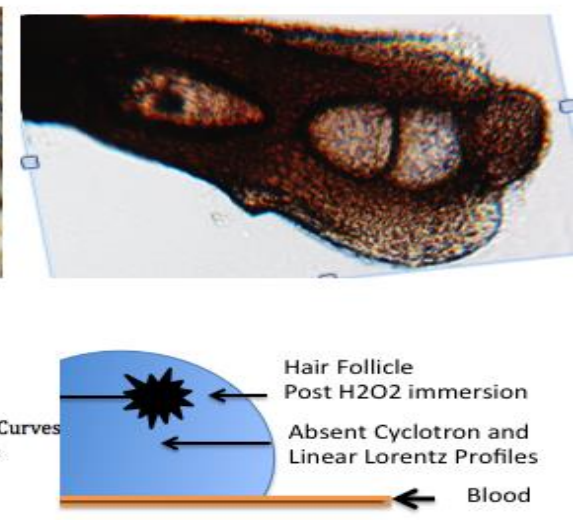

D

Figure 1B: Slide assembly showing A= Fresh Living Hair Follicle. B=Follicle post $\mathrm{H} 2 \mathrm{O}$ immersion. $\mathrm{C}=$ Computer diagram showing slide assembly with live follicle showing MPs DComputer diagram showing dead follicle and Absence Of MPs.

The hair follicle was then transferred to a clean slide and covered with drops of $35 \% \mathrm{H} 2 \mathrm{O} 2$. Under video surveillance, when the catalase ceased to decompose the $\mathrm{H} 2 \mathrm{O} 2$ the hair was transferred to the slide assembly and the experiment duplicated as in the baseline experiments.

\section{Results}




\section{Moving Blood and Moving linear Lorentz Forces}

In order to display moving blood in the SDW, speed is the essence. The hair is placed on a slide and covered with water, drops of blood are placed on a second slide, the SDW is completed with the first slide and placed on the microscope viewing platform, focused and video-recorded as the focusing knob is slowly moved inwards. For a visual display, the Linear Lorentz Forces are chosen. The figure below documents a static image of the displayed linear Lorentz Forces moving in tune with the moving trapped blood. This is best duplicated seen in the first $10 \mathrm{sec}$ of the blood drops trapping. (Fig 2 and video link: https://youtu.be/UUFwGfVoXbw)

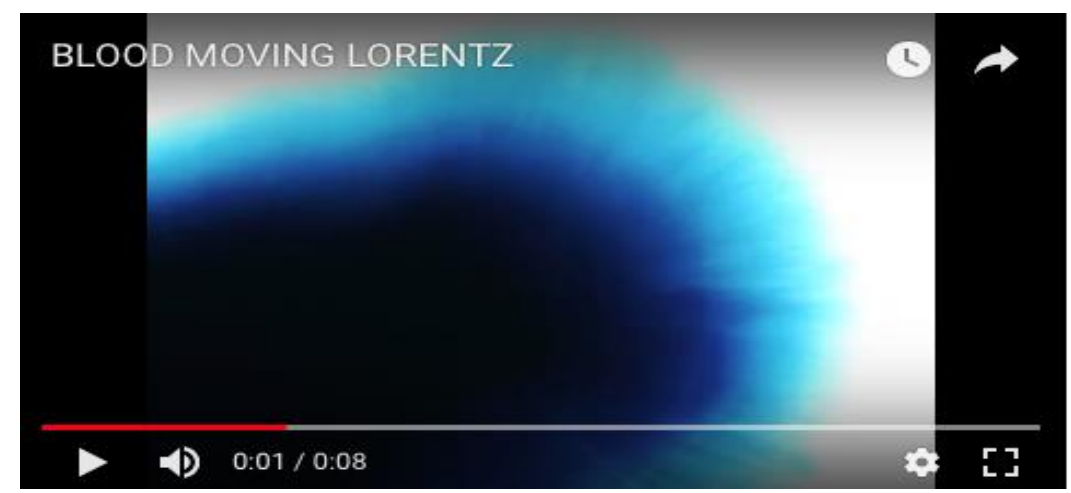

Figure 2: Microphotograph of video frame depicting moving linear Lorentz Forces in synchrony with moving blood trapped in two glass slides. Video link:

https://youtu.be/UUFwGfVoXbw

\section{Static Images of Mps}

The first MPs were identified as having a curved cyclotron resonance pattern (Fig 3) below:

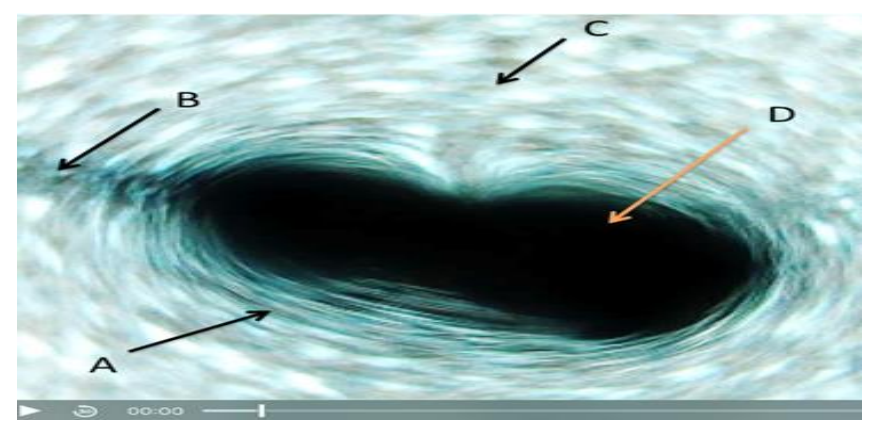

Figure 3: Demonstration of human blood magnetic properties. Microphotograph of a still video frame of fresh moving (expanding field) human blood immediately after SDW was prepared.

Seen on top slide is a human hair follicle and shaft covered by distilled water. This image shows circumferential lines as a product cyclotron resonance, which is caused by curving or charges in

a magnetic field as the electrical field changes polarity across the conductive bodies. $\mathrm{A}=$

Circumferential lines $\mathrm{B}=$ Hair shaft $\mathrm{C}=$ Sandwiched moving blood smear $\mathrm{D}=$ Hair follicle. Duplicated from: Embi AA. Human Blood Magnetic Image reproduced from: Embi AA. Human Blood Magnetic Profiles Interactions: Role in Mosquito Feeding. Journal of Nature and Science (JNSCI), 2(3): e186, 2016.) 
And as the knob moved inwards, the second MPs were also identified this time as linear Lorentz Forces as result of the Hall effect. (Fig 4).

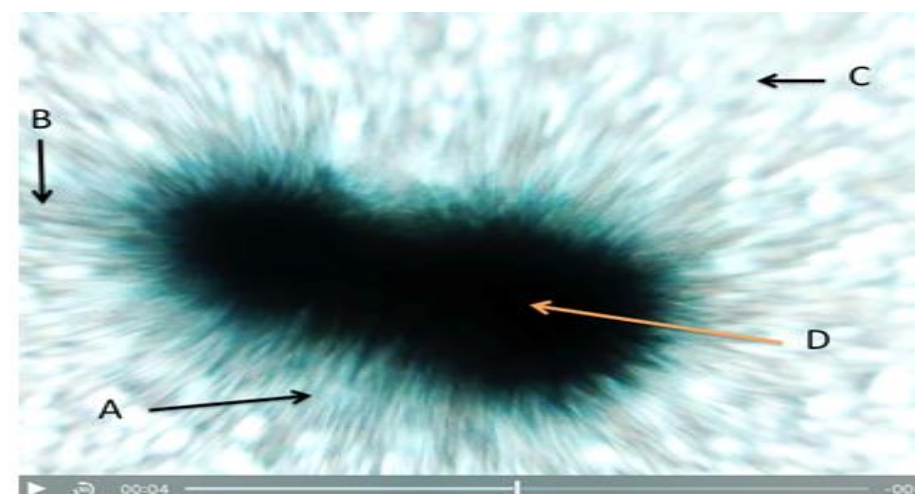

Figure 4: Focusing knob moved inwards. Microphotograph of video frame of hair follicle and shaft on top of blood SDW. Both hair and blood maintain magnetic profiles and are piezoelectric ie: convert oscillations to mechanical vibrations and vice versa. $A=$ Linear extensions viewed represent Lorentz forces as a result of the Hall effect. $B=$ Hair shaft. $C=$ Out of focus blood in SDW. $\mathrm{D}=$ Hair follicle. (Please see supplemental video recordings showing movement of the Lorentz forces as the blood settles in the SDW: Image reproduced from: Embi AA. Human Blood Magnetic Profiles Interactions: Role in Mosquito Feeding. Journal of Nature and Science (JNSCI), 2(3): e186, 2016.)

\section{Multiple MPs foci seen in Control Hairs}

An additional finding was the identification of several MPs foci found in the same hair sample. This is shown in Figure 5 and video https://youtu.be/xHAAjB_DGDI

This phenomenon has not been previously described, and its interpretation is unknown at present.

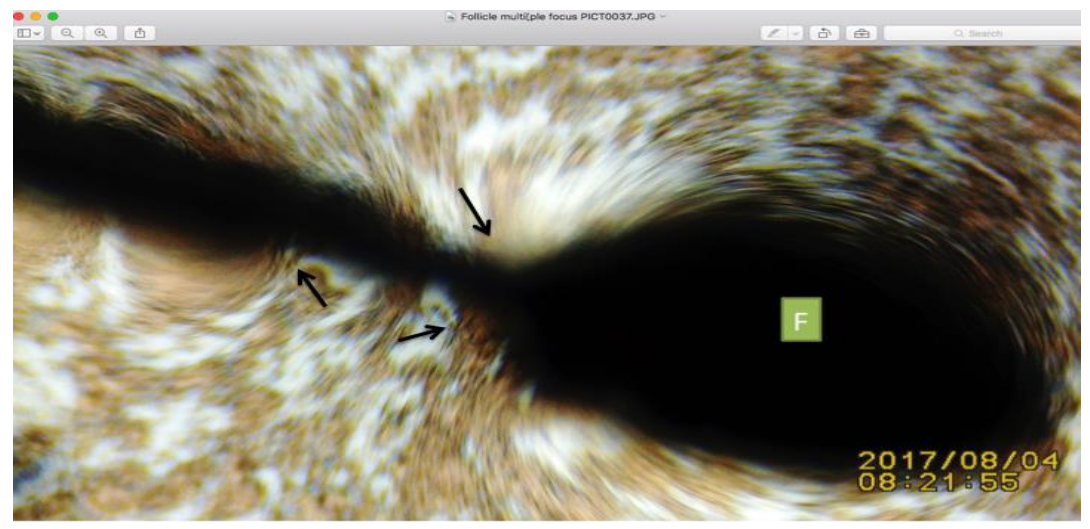

Figure 5: Freshly plucked Follicle in Anagen- F= Follicle. Arrows showing different foci of

Magnetic Profiles resembling linear Lorentz Forces and Cyclotron Resonance curved lines Please link https://youtu.be/xHAAjB_DGDI for additional details.

\section{Control experiments in the absence of blood in the SDW.}

In all experiments $(n=3)$ performed in the absence of blood in the SDW, the MPs images could not be reproduced. This is shown in Fig 6 below and video recording https://youtu.be/MvLKHWbcIuI 


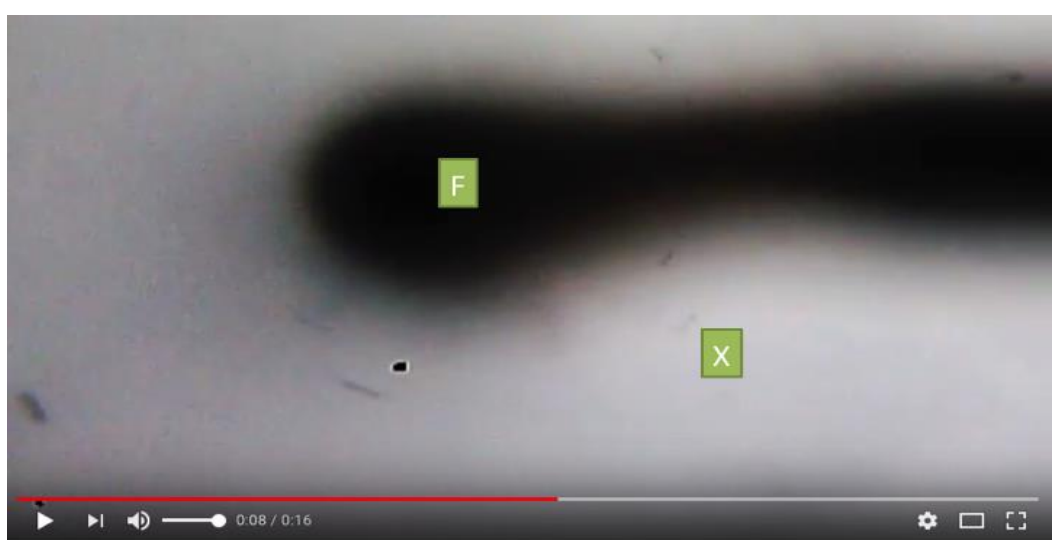

Figure 6: Microphotograph of out of focus hair follicle in slide assembly. Pure water substituted blood in SDW. There is an absence of magnetic profiles

For details link to video recording: https://youtu.be/MvLKHWbcIuI

\section{Hairs Exposed to $\mathrm{H} 2 \mathrm{O} 2$}

After the baseline MPs were recorded, the hairs were then transferred and placed on a clean slide and covered with drops of $35 \% \mathrm{H} 2 \mathrm{O} 2$. After the $\mathrm{H} 2 \mathrm{O} 2$ decomposition ceased, the hairs were rinsed in distilled water and placed again on the slide assembly. Recordings were made attempting to image MPs.

\section{Example of Hair Follicle immediately after H2O2 exposure (fig 7)}

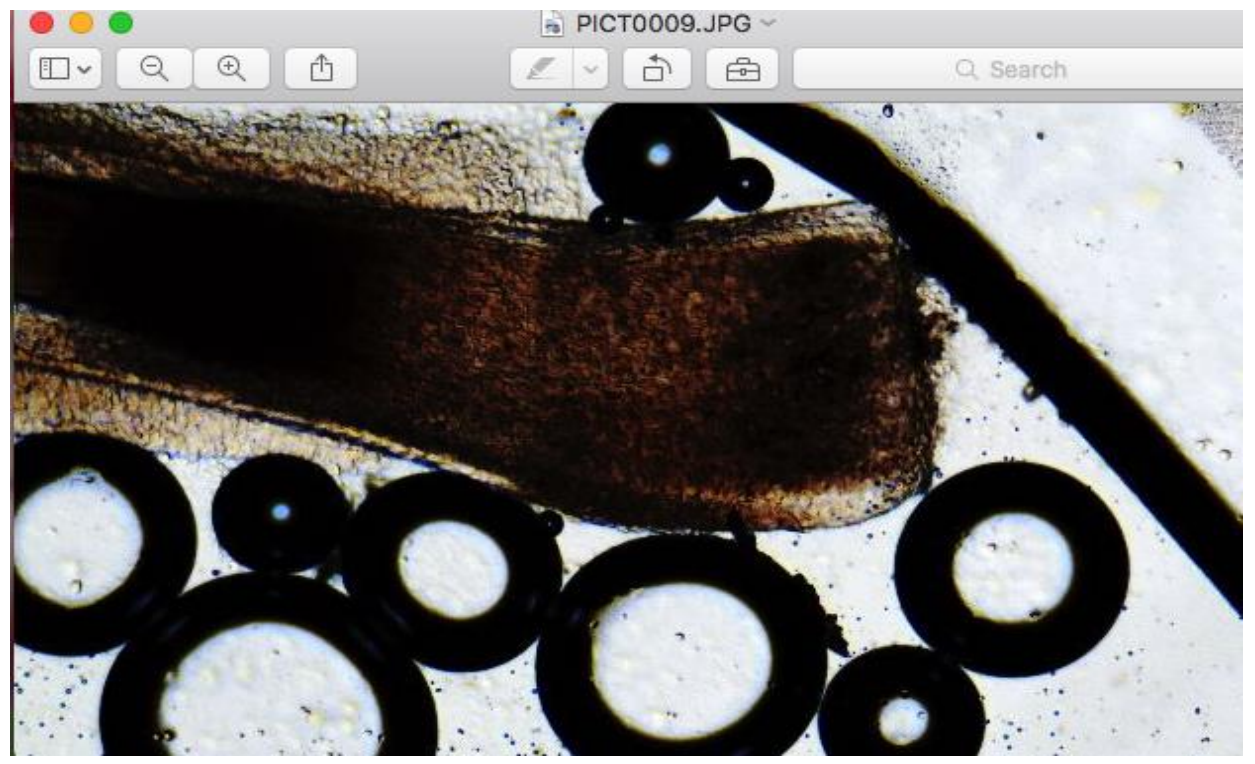

Figure 7: Control hair follicle in $35 \% \mathrm{H} 2 \mathrm{O} 2$. Notice the release of oxygen bubbles indicating $\mathrm{H} 2 \mathrm{O} 2$ molecules breakdown by Catalase

\section{Hair Follicles Macroscopic Appearance post $\mathrm{H2O2}$}

In contrast with the above figure, after 2 hours the destructive effect of $35 \% \mathrm{H} 2 \mathrm{O} 2$ on the hair follicle is shown below (Figs 8,9) 


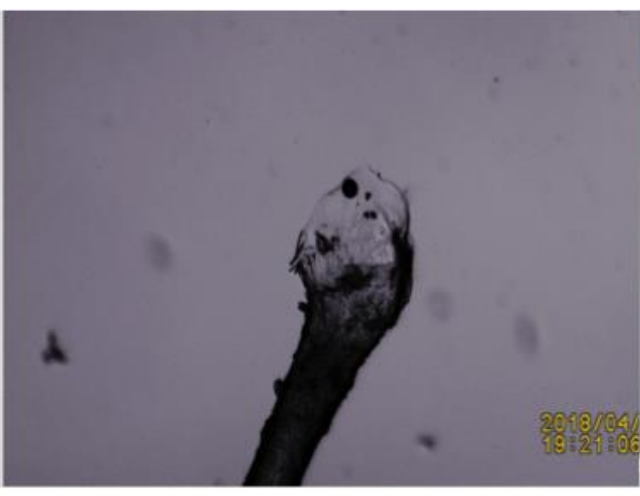

A

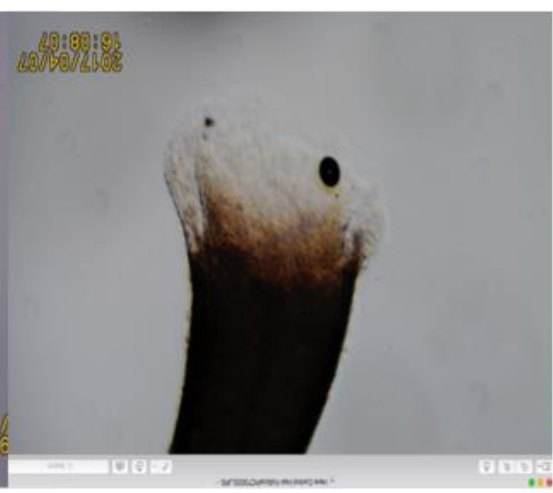

B

Figure 8: Panel $\mathrm{A}=$ Hair Follicle post dipping in 35\% H2O2. Panel $\mathrm{B}=\mathrm{Control}$ Hair. Notice the tissue shrinkage seen macroscopically in the treated sample

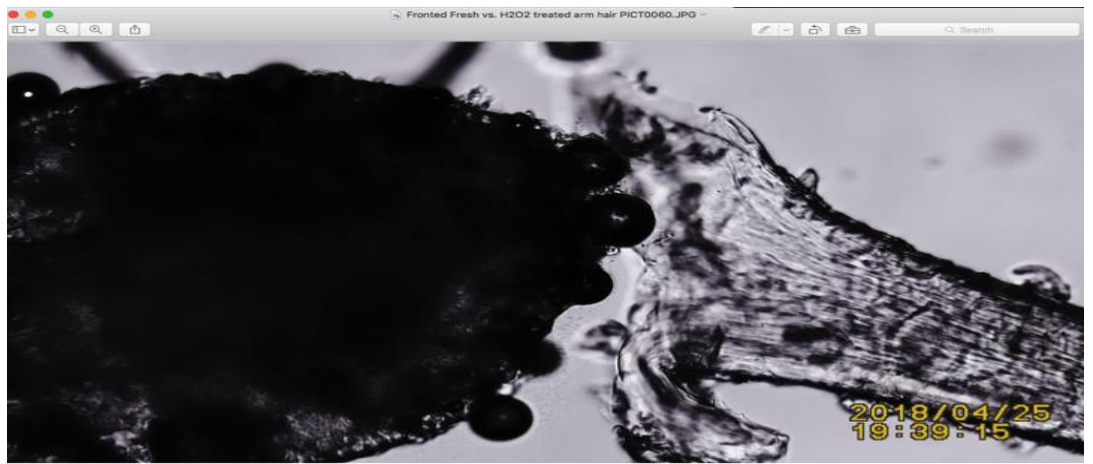

Figure 9: Another example of a freshly plucked arm hair follicle (left) facing catalase-depleted sample (right). Notice the $\mathrm{H} 2 \mathrm{O} 2$ decomposition (oxygen bubbles) in the fresh sample (left image). The $\mathrm{H} 2 \mathrm{O} 2$ treated follicle (right side) is deformed, dehydrated and dead, as unable decompose $\mathrm{H} 2 \mathrm{O} 2$ molecules

Please refer to video link for details: https://youtu.be/x5ux3uf_EX0

\section{Dynamic Example of Lack of Magnetic Profiles Due to Catalase Depletion}

As shown in (Fig 10) below, After post $\mathrm{H} 2 \mathrm{O} 2$ treatment the hair follicle is unable to produce Magnetic Profiles $(n=12)$ as visually demonstrated in https://youtu.be/MCR5W76eqe8

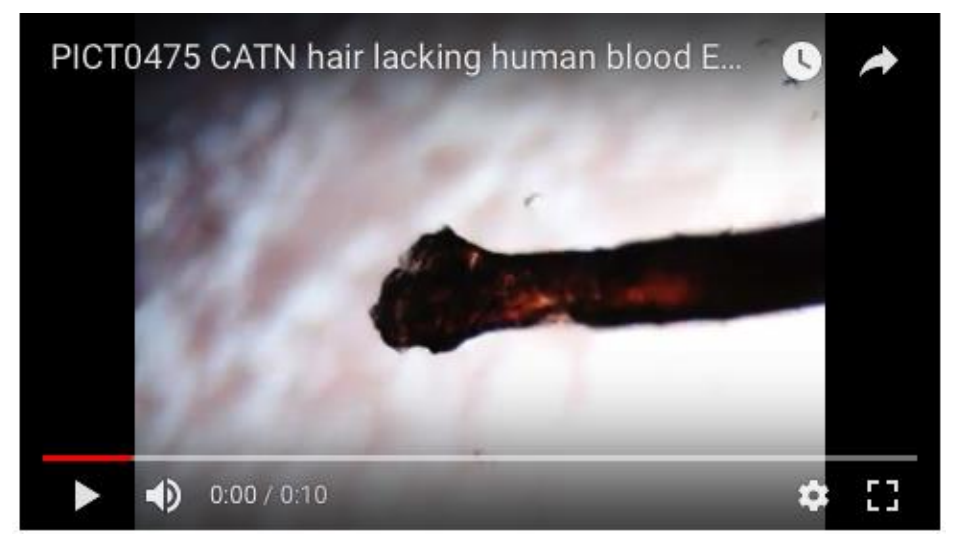

Figure 10: Arm hair follicle post $\mathrm{H} 2 \mathrm{O} 2$ treatment. Microphotograph from Video-recording showing $X=$ Sandwiched between two glass slides of drops of blood 
The video recording at this stage shows absence of Magnetic Profiles (MPS). See link https://youtu.be/MCR5W76eqe8 Shows post H2O2 treatment unable to produce Magnetic Profiles. Compare with Figure 6 and link https://youtu.be/xHAAjB_DGDI

\section{Discussion}

The metabolic process of hair follicles, blood and biological active organisms, maintain magnetic profiles and are piezoelectric ie: convert oscillations to mechanical vibrations and vice versa. The attraction of a magnetic substance to a body part must be based upon electromagnetic interaction. The basis is always piezoelectricity or electromechanical transduction. There is a possible explanation for the mechanism causing the spatial MPs images presented. Is theorized that the intrinsic endogenous BMFs emitted during cell respiration caused by the $\mathrm{H}_{2} \mathrm{O}_{2}$ breakdown by the ubiquitous protein enzyme catalase (13) present in the living follicle could interact with the "magnetic properties of oxy- and deoxyhemoglobin, as well as those of closely related compounds containing hemes" (14). The water drops serving as a medium for the display of the magnetic particles.

\section{The Role of Resonance Signaling in Medicine}

From a Biophysics perspective, resonance signaling has been identified as a potential tool in regulating biological information $(15,16)$, and regenerative medicine. This manuscript introduces a positive correlation between Resonance Signaling and Life. As a corollary the absent of resonance signaling indicates tissue death.

\section{Acknowledgements}

The author acknowledges the expertise of Mrs. Laura Sanchez Embi for the graphic designs. Also, the invaluable contribution for images interpretation by the renowned physicist Professor Dr. Jerry I. Jacobson Chief Science Officer at Pico-Tesla Magnetic Therapies. Also acknowledged is Benjamin J. Scherlag PhD from the University of Oklahoma, Health Sciences Center USA for the supply of pure water.

\section{References}

[1] Schneider MR, Schmidt-Ullrich R, Paus R. The hair follicle as a dynamic miniorgan. Curr Biol. 2009 Feb 10;19(3): R132-42. doi: 10.1016/j.cub.2008.12.005.

[2] Baule G.M, McFee R. Detection of the magnetic field of the heart. American Heart Journal 1963;66: 95-96 PBMID: 14045992.

[3] Cohen D. Magnetoencephalography: Detection of the Brain's electrical activity with a superconducting magnetometer. Science 1972;175: 664-666 PMID: 5009769.

[4] Cohen D, Kaufman LA. Magnetic determination of the relationship between the ST segment shift and the injury current produced by coronary occlusion. Circ Res 1975;36: 414-424 PMID: 1111998.

[5] induced S-T segment depression. Circ Res 1983;53: 274-279 PMID: 6883650.

[6] Cohen D, Palti Y, Cuffin BN, Schmid SJ. 1980. Magnetic fields produced by steady currents in the body. Proc. Natl. Acad. Sci. USA; 77(3): 1447-1451. 
[7] Benjamin J. Scherlag, Kaustuv Sahoo, Abraham A. Embi A. 2016 Novel and Simplified Method for Imaging the Electromagnetic Energy in Plant and AnimalTissues. Journal of Nanoscience and Nanoengineering Vol. 2, No. 1, pp. 6-9

[8] Demonstration of Inherent Electromagnetic Energy Emanating from Isolated Human Hairs. 2015. Abraham A. Embi, Jerry I. Jacobson, Kaustuv Sahoo, Benjamin J. Scherlag, JNSCI 1(3):e55.

[9] Abraham A. Embi, Benjamin J. Scherlag Demonstration of Human Hair Follicle Biomagnetic Penetration Through Glass Barriers International Journal of Materials Chemistry and Physics Vol. 2, No. 2, 2016, pp. 71-74

[10] Scherlag BJ, Sahoo K (2016) Electromagnetic Imaging of Subdermal Human Hair Follicles InVivo. J Nat Sci, 2(2):e174.

[11] Abraham A. Embi1, Benjamin J. Scherlag. Demonstration of Biomagnetic Responses of Paired Human Hair Follicles Using Nano-sized Iron Particles Solutions: Inhibition of Diamagnetic Crystallization International Journal of Materials Chemistry and PhysicsVol. 2, No. 2, 2016, pp. 84-87

[12] Bren KL, Eisenberg R, Gray HB Discovery of the magnetic behavior of hemoglobin: A beginning of bioinorganic chemistry. Proc Natl Acad Sci U S A. 2015 Oct 27;112(43):13123-7. doi: 10.1073/pnas. 1515704112.

[13] Embi AA. Endogenous electromagnetic forces emissions during cell respiration as additional factor in cancer origin. Cancer Cell International.

[14] Bren KL, Eisenberg R, Gray HB Discovery of the magnetic behavior of hemoglobin: A beginning of bioinorganic chemistry. Proc Natl Acad Sci U S A. 2015 Oct 27;112(43):13123-7. doi: 10.1073/pnas. 1515704112 .

[15] Foletti, Alberto \& Grimaldi, Settimio \& Lisi, Antonella \& Ledda, Mario \& Liboff, Abraham. (2013). Bioelectromagnetic medicine: The role of resonance signaling. Electromagnetic biology and medicine. 32. 10.3109/15368378.2012.743908.

[16] Lisi A, Ledda M, de Carlo F, Pozzi D, Messina E, Gaetani R, Chimenti I, Barile L, Giacomello A, D'Emilia E, Giuliani L, Foletti A, Patti A, Vulcano A, Grimaldi S. Ion cyclotron resonance as a tool in regenerative medicine. Electromagn Biol Med. 2008;27(2):127-33.

doi: 10.1080/15368370802072117.

*Corresponding author.

E-mail address: embi21@att.net 